\title{
Modular Optofluidic Systems (MOPS)
}

\author{
Tobias N. Ackermann ${ }^{\mathrm{a}}$, Jiri Dietvorst ${ }^{\mathrm{a}}$, Ana Sanchis ${ }^{\mathrm{b}, \mathrm{c}}$, Juan P. Salvador ${ }^{\mathrm{b}, \mathrm{c}}$, Xavier Munoz-Berbel ${ }^{\mathrm{a}}$, \\ Erica Alvarez-Conde ${ }^{a}$, Daniel Kopp ${ }^{d}$, Hans Zappe ${ }^{\mathrm{d}}$, M.-Pilar Marco ${ }^{\text {b,c }}$, Andreu Llobera ${ }^{* a}$ \\ anstitut de Microelectrònica de Barcelona (IMB-CNM, CSIC), Campus UAB, Barcelona, Spain; \\ ${ }^{\mathrm{b}}$ Nanobiotechnology for Diagnostics (Nb4D), IQAC-CSIC, Barcelona, Spain; ${ }^{\mathrm{C}} \mathrm{CIBER}$ de \\ Bioingeniería, Biomateriales y Nanomedicina (CIBER-BBN), Spain; ${ }^{\mathrm{d}}$ Gisela and Erwin Sick Chair \\ of Micro-optics, Department of Microsystems Engineering (IMTEK), University of Freiburg, \\ Georges-Köhler-Allee 102, 79110 Freiburg, Germany.
}

\begin{abstract}
Elementary PDMS-based building blocks of fluidic, optical and optofluidic components for Lab on a chip (LOC) platforms has here been developed. All individual modules are compatible and can be anchored and released with the help of puzzle-type connectors This approach is a powerful toolbox to create modular optofluidic systems (MOPS), which can be modified/upgraded to user needs and in-situ reconfigurable. In addition, the PDMS can locally be functionalized, defining a modular biosensor. Measurements in absorbance and fluorescence have been pursued as demonstrator.
\end{abstract}

Keywords: PDMS, modular, Photonic Lab on a chip, Medical optics instrumentation, Spectroscopy, fluorescence and luminescence, Absorption

\section{INTRODUCTION}

Lab-on-a-Chip (LoC) technology has gained significant weight in the last two decades, enabling the miniaturization of an analytical assay, with the resulting decrease of the required analysis time, sample volume and reagent consumption. Even more importantly, it allows the enhancement of the sensitivity and the possibility to parallelize the analysis without dramatically increasing both the complexity and the system footprint. As a result, from the early Micro Total Analysis System ( $\mu \mathrm{TAS}$ ) concept [1] nowadays it is possible to find in the literature a myriad of extremely sophisticated LoC for applications such as drug development [2], genomics [3] clinical diagnosis [4] or cellomics.[5].

Even though the evolution of the LoC concept has progressed dramatically, they still require external instrumentation for analyte detection and quantification. As a result, their entrance to the market is still limited. Steps towards addressing this issue have already been taken with the integration of transduction mechanisms in the LoC concept, such as electrochemical [6], magnetic [7] or photonics [8] Among them, the synergistic combination of integrated photonic elements with LoC gives rise to the Photonic Lab-on-a-Chip (PhLoC) concept $[9,10]$. Here, the main function of microfluidics is the manipulation and transport of the analytes, while the photonic elements transduce the (bio)chemical signal arising from the analytes in-situ to a quantifiable signal.

The above mentioned exemples of LoCs and PhLoCs are monolithic, with all required elements integrated in a system. Alignment is inherently provided by the mask aligners, while the facet quality is given by the type of masks used. Even though monolithic $(\mathrm{Ph})$ LoCs can be considered as the configuration with the highest robustness, its main disadvantage is that a single defect on the causes a complete malfunction of the overall system. This is especially critical in complex $(\mathrm{Ph}) \mathrm{LoCs}$ that require either long processing times or complex LoC-to-world interconnection. Additionally, it must be considered that for each application, $(\mathrm{Ph}) \mathrm{LoCs}$ require an optimized geometry that is normally achieved by recursive iterations, requiring thus an entire new fabrication cycle, which goes against the low cost concept of the $(\mathrm{Ph}) \mathrm{LoC}$.

Even requiring a specific configuration, it is also true that all LoC share common basic elements such as inlet/outlets, channels or flow dividers and, in the case of PhLoCs, microoptics, photonics or fiber optics alignment channels. In this context, an alternative approach is to define a set of elementary fully compatible building blocks that include the different required elements that can be optimized separately before integration into a larger system in a modular fashion. 
In this approach, a malfunction of a given module can be tackled by its direct replacement without affecting the overall behavior of the system. This concept has already been explored using pre-fabricated microfluidic assembly blocks (MABs) [11] and modular architectures utilizing fluidic breadboards [12]. Surprisingly, this modular approach suffers from the same weak point as the monolithic LoC, namely the off-chip transduction, thus requiring complex instrumentation for analyte detection and quantification. As a result, they still fail to be more widely established alongside standardized laboratory protocols and techniques and, more importantly, in real applications outside controlled laboratory environment. A first attempt towards implementing photonic detection in a LoC has been done by defining the so-called modular optoelectronic microfluidic backplane [13] which, even extremely promising, requires expensive fabrication steps. Additionally, each backplane is highly specific, resulting in low assembly flexibility.

Reduction of fabrication costs are associated with polymers and simple fabrication techniques. Among them, poly(dimethylsiloxane) (PDMS) and soft lithography [14] are excellent examples, respectively. PDMS has been widely applied in the development of LoC due to its fabrication simplicity and properties, such as low Young's modulus (300$800 \mathrm{KPa})$, a low refractive index $(\mathrm{n}=1.41)$ and transparent in the UV-NIR range. Albeit, its hydrophobic character makes this material very prone to serious non-specific absorption processes of different organic molecules and biomolecules when applied to biochemical analysis. To tackle this issue, several surface modification processes have been reported to obtain silanol groups $(\mathrm{Si}-\mathrm{OH})$ on the material surface and allow tuning its hydrophilic/hydrophobic balance [15]. Even valid, this approach is reversible, and therefore PDMS recovers its hydrophobicity with time. An alternative is to introduce hydroxyl (-OH) groups on the PDMS surface by physisorption of polyvinyl alcohol (PVA) polymer [16] and further silanization [17] of the resulting surface. At this stage, functional groups can be introduced enabling the covalent attachment of biomolecules [18], finally providing to the LoC the desired selectivity for specific analyte while minimizing interference due to non-specific adsorption of other analytes.

As a proof of concept, the development of a LoC device based on a Modular Optofluidic System (MOPS) with colorimetric detection was carried out for the detection of CRP. C-reactive protein (CRP) is one of the established biomarkers of inflammation that are related for the early diagnosis of certain cardiovascular diseases (CVDs). Even if it was not considered a useful clinical parameter for many years because of its unspecificity, nowadays it is measured as a sensitive systemic marker of inflammation, infection and tissue damage and moreover it has emerged as an interesting novel and potentially clinically useful marker for increased cardiovascular risk [19]. According the National Academy of Clinical Biochemistry (NACB) recommended CRP as the inflammatory biomarker of choice for assessment of cardiovascular risk [20]. Therefore, there is an unmet need for the monitorization of the CRP simple, rapid and relatively inexpensive way for reducing hospital stay, complications and improving adherence to treatment [21]

The combination of specific biofunctionalization with modular assembling blocks provides nothing but advantages in terms of flexibility, specificity and working simplicity. Given an arbitrary modular LoC configuration, different off-chip biofunctionalized blocks could be sequentially or in parallel inserted, obtaining its response against a given target analye. As above discussed, in case of malfunction or for preserving the biofunctionalization, the building block can be removed from the system and either discarded or stored in adequate environment. Despite such outstanding approach, to date there has been no contribution regarding full reconfigurable, interchangeable and compatible modular optofluidic systems able to assemble arbitrarily complex PhLoCs. This open issue is addressed in this work.

\section{DESIGN AND FABRICATION}

The Modular Optofluidic System (MOPS) shown in Figure 1 consists of several fully compatible modules, which aim to cover most of the basic functions of any PhLoC. Each module exhibits plugs and sockets for reciprocal anchorage, thus ensuring optimal alignment and tight interconnections. Module I comprises a corrugated blind channel where 230 $\mu \mathrm{m}$ fiber optics can be inserted. The end of this channel matches with the focal point of the microlens, which has been defined only using PDMS and air. When the finer optics is position at the focal point of this microlens, the numerical aperture of the fiber optics is corrected, having then parallel beams at the output of Module I. Module IIa has an optofluidic configuration, comprising two air mirrors [22] enabling the definition of PDMS lightguides. Light confined is coupled to a microfluidic channel (in green in Figure 1) also having air mirrors at both sides to minimize optical losses. Module IIb is complementary to IIa. Module III is a fluidic path extension. Module IV comprises a microfluidic channel able to be filled with a specific colorant which absorbs specific wavelengths, thus defining an absorbance filter. It also includes two lightguides at the input/output of this module. Modules V and VI correspond to the fluidic inlet and outlets respectively. The main difference between them is that Module $\mathrm{V}$ includes an internal pressure controller, comprising a $\mathrm{V}$-shape structure where an air bubble is entrapped. 
Fabrication is based on soft lithography and replica molding. Masters are fabricated by two-level SU-8 technology, in a similar approach as presented in [23]. The first level defined the optofluidic structures, whereas the second defined the frame and allowed the definition of the plugs and sockets. A second master was also defined having only the frame, which defines the bottom part of the modules. Once the masters were defined, The PDMS pre-polymer was obtained by mixing the curing agent with the elastomer base in a 1:10 ratio (v:v). The resulting mixture was subsequently degassed to remove air bubbles. Pouring over the masters was done carefully to avoid overflow and subsequently cured for 20 minutes at $80^{\circ} \mathrm{C}$. After peeling off the two PDMS sections, they were exposed to an oxygen plasma in a barrel etcher (Surface Technology Systems, Newport, UK). Immediately afterwards, both sections are brought in contact using DI water as lubricant. Alignment was done with the help of a microscope. Once aligned, they were located at $80^{\circ} \mathrm{C}$ for $1 \mathrm{~h}$, causing its irreversible sealing and finishing the fabrication of the modules.

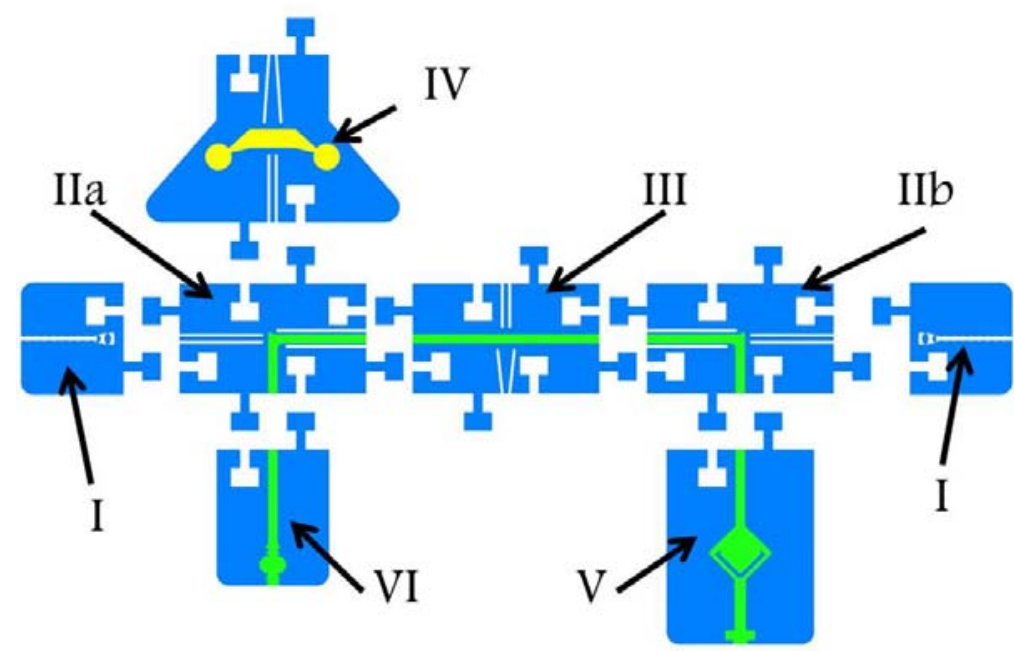

Figure 1: MOPS for absorbance and/or fluorescence measurements: module I clamps a fiber optics. Light is coupled to IIa, which is transferred to a microchannel. Module II is a microchannel with a length of $1 \mathrm{~cm}$. IIb brings the light which has interacted with the liquid at the microchannel to an output fiber optics, again located in a module type I. An xerogel absorbance filter (IV) [24] filter excitation wavelengths when measuring fluorescence. The fluidic inlet module (V) has an air-bubble based internal pressure regulator, which allows to obtain leak-free MOPS, fluid is finally collected with the outlet module (VI).

A finished example of an absorbance-based MOPS can be seen in figure 2, where all the previously discussed modules are used except III and IV. It was filled with crystal violet for visualization. It can be seen that the fabricated MOPS are leak-free and that the air bubble at the fluidic inlet defines the internal pressure controller.

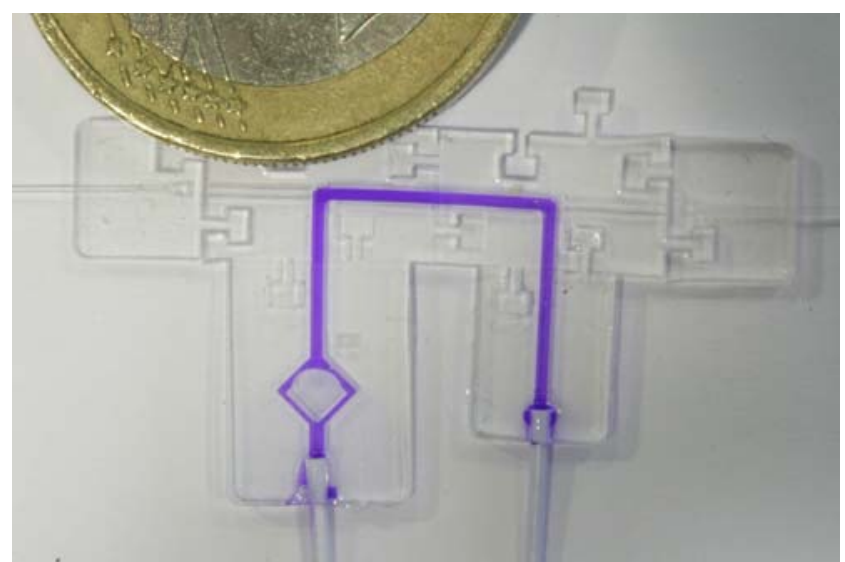

Figure 2: Image of the MOPS for absorbance/fluorescence filled with Crystal Violet for visualization. 


\section{IMMUNOCHEMICAL ASSAY}

The immunochemical assay consisted in sandwich immunoassay, using both anti-CRP antibodies as a capture and biotinylated detection antibody, and the signal was acquired by the addition of and Streptavidin-HRP conjugated using $\mathrm{H} 2 \mathrm{O} 2 / \mathrm{TMB}$ as the colored substrate. The method of immobilization was previously tested [18] resulting the PVA coating surface the optimum for the incorporation of biomolecules onto the PDMS surfaces. The method was modified with slightly modifications. All the solutions and liquids were pumped using a perfusion pump at the corresponding flow rate (model Fusion 200, Chemyx Inc., Stafford, TX). PDMS surfaces were cleaned with ethanol $(1 \mathrm{~mL})$ and ultrapure water $(1 \mathrm{~mL})$ at $100 \mu \mathrm{L} / \mathrm{min}$. The modification of the PDMS was performed pumping a solution of PVA at $1 \mathrm{mg} / \mathrm{mL}(1$ $\mathrm{mL})$ at $50 \mu \mathrm{L} / \mathrm{min}$. Afterwards, the surfaces were cleaned with ultrapure water $(1 \mathrm{~mL})$ at $100 \mu \mathrm{L} / \mathrm{min}$. The functionalization of the surface was performed pumping a solution of Glycidoxypropyltrimethoxysilane (GPTMS), 2.5\% in freshly destilled ethanol $(1 \mathrm{~mL})$ at $50 \mu \mathrm{L} / \mathrm{min}$. The surfaces were cleaned with ethanol and dried with nitrogen. Inmediatly, a solution of $100 \mu \mathrm{g} / \mathrm{mL}$ of the AbCRP2 capture antibody in PBS $(10 \mathrm{mM}, \mathrm{pH}=7.5)(1 \mathrm{~mL})$ were pumped into the MOPS at $20 \mu \mathrm{g} / \mathrm{mL}$. Then, the chips were rinsed with PBST $(1 \mathrm{~mL}, 10 \mathrm{mM}, 0.05 \%$ Tween $20, \mathrm{~m} \mathrm{pH} 7.5)$ at 100 $\mu \mathrm{L} / \mathrm{min}$. The CRP at different concentrations were prepared in PBST and then introduced into the MOPS chips $(1 \mathrm{~mL})$ at $50 \mu \mathrm{L} / \mathrm{min}$. Then, the chips were rinsed with PBST at $100 \mu \mathrm{L} / \mathrm{min}$. A solution of the biotynilated detection antibody AbCRP1-B at $1 \mu \mathrm{g} / \mathrm{mL}$ in PBST $(1 \mathrm{~mL})$ at $50 \mu \mathrm{L} / \mathrm{min}$. Then, the chips were rinsed with PBST at $100 \mu \mathrm{L} / \mathrm{min}$. A solution of streptavin-HRP conjugate at $1 \mu \mathrm{g} / \mathrm{mL}$ in PBST $(1 \mathrm{~mL})$ at $50 \mu \mathrm{L} / \mathrm{min}$ was introduced into MOPS chips. Then, a citrate solution ( $1 \mathrm{~mL}, 0.05 \mathrm{M}, \mathrm{pH}=5.5$ ) was pumped through the chip at $50 \mu \mathrm{L} / \mathrm{min}$ and then, a substrate solution containing $0.01 \% \mathrm{TMB}$ (tetramethylbenzidine) and $0.004 \% \mathrm{H}_{2} \mathrm{O}_{2}$ in citrate buffer. Two different chips were functionalized. In one side, a sample spiked at $10 \mathrm{ng} / \mathrm{mL}$ of CRP in PBST was pumped into a chip, and, on the other hand, a second functionlized chip was used as a control, pumping just PBST.

\section{CHARACTERIZATION}

For absorbance measurements, the MOPS was assembled without the absorbance filter module IV. Assembly of modules IIIa and IIIb provides with an optical path of $1 \mathrm{~cm}$. Different Quinoleine Yellow concentrations (ranging from 5 to $500 \mu \mathrm{M}$ ) were successively injected in the module V (outlet is module VI). Calibration was done coupling a $230 \mu \mathrm{m}$ fiber optics to a broadband light source. Readout comprised an identical fiber optics and a spectrophotometer (QE65000 pro, Ocean Optics). For Fluorescence measurements using Fluorescein as analyte, the broadband light source was replaced by a $405 \mathrm{~nm}$ diode laser (Micos $\mathrm{GmbH}$, Germany). The absorbance filter module was incorporated into the optical path to block the excitation wavelength, which was coupled to the input fiber optics in two configurations $\left(180^{\circ}\right.$ and $90^{\circ}$ ).

\subsection{Fluorescence measurements}

Fluorescein diluted in PBS was used as target analyte with concentrations ranging from 10 to $75 \mu \mathrm{M}$. The MOPS comprised all the modules above described except III, since the fluorescence signal does not depend on the optical path. A $405 \mathrm{~nm}$ diode laser (Micos GmbH, Germany) was coupled to a $230 \mu \mathrm{m}$ fiber optics. Readout comprised an identical fiber optics and a spectrophotometer (QE65000 pro, Ocean Optics). For defining the absorbance filter, Quinoleine Yellow was mixed with a sol solution following the protocol described in [24], just afterwards, it was injected in the microfluidic element included in the absorbance filter module. After drying, the resulting sol-gel showed no cracks and no colorant bleaching. This module was then incorporated into the optical path to block the excitation wavelength in two configurations $\left(180^{\circ}\right.$ and $\left.90^{\circ}\right)$. Figure $3 \mathrm{a}$ is used as reference and shows the results in $180^{\circ}$ configuration without using the filter module. Including the filter module (Figure $3 \mathrm{~b}$ ) allows a significant enhancement of the measured signal. Finally, in the optimal configuration for fluorescence $\left(90^{\circ}\right)$, a 2 -fold performance enhancement. 


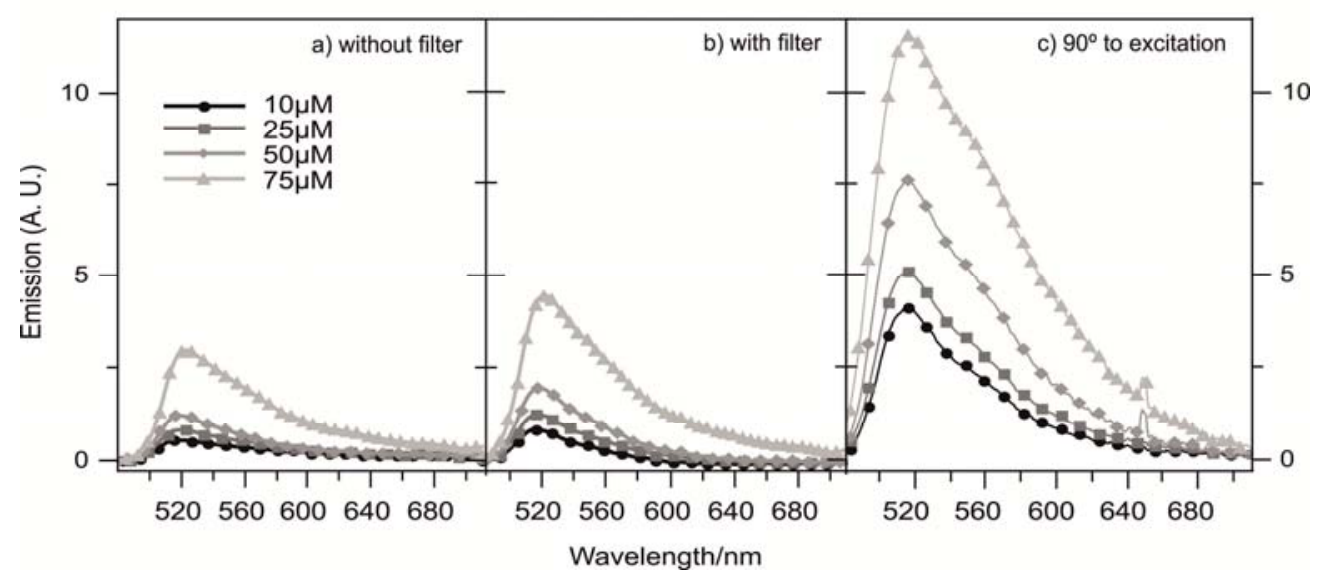

Figure 3: Emission-spectra in the three MOPS configurations studied (no filter, filter + readout at $180^{\circ}$ and filter + readout at $90^{\circ}$ ). The measurement sensitivity is increased when incorporating the filter module.

\subsection{Absorbance measurements}

For absorbance measurements, the MOPS was assembled without the absorbance filter module. Assembly of modules IIIa and IIIb provided with an optical path of $1 \mathrm{~cm}$, which was at a later stage extended to $2 \mathrm{~cm}$ when the module II was included. Different Quinoleine yellow concentrations (ranging from 5 to $500 \mu \mathrm{M}$ ) were successively injected in the module V (outlet is module VI). Calibration was done coupling a $230 \mu \mathrm{m}$ fiber optics to a broadband light source. Readout was identical to the fluorescence measurements. Figure 4 shows the results obtained with the two different lengths. As expectable, the MOPS with an optical path had a larger linear range (from 0 to $100 \mu \mathrm{M}$ ), and a Limit of Detection (LoD) of $5 \mu \mathrm{M}$. The inclusion of module III resulted in a shortening of the linear range (from 0 to $50 \mu \mathrm{m}$ ), even though the LoD decreased to $3.9 \mu \mathrm{M}$ and the sensitivity increased a factor 1.7 , which nearly matches the expected factor 2 considering that the optical path was doubled.

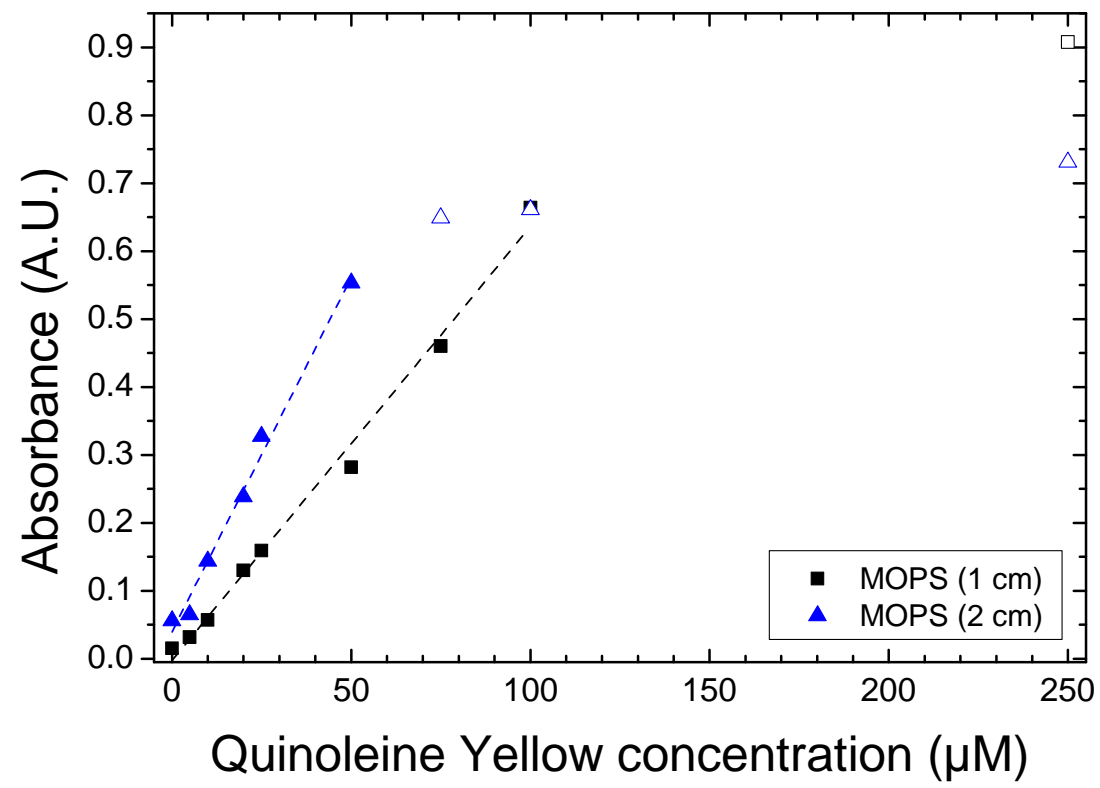

Figure 4: Absorbance as a function of the Quinoleine Yellow concentration for a MOPS assembled for absorbance. Initially the optical path was fixed to $1 \mathrm{~cm}$. Inclusion of Module III expanded this path to $2 \mathrm{~cm}$. 


\subsection{Biofunctionalized absorbance measurements}

The method of conjugation resulted the most suitable method for the immobilization of the detection antibody, as it was discussed in a previous work [18]. The method consisted in the derivatization of the hydrophobic PDMS with a hydrophilic polymer such as PVA. This hydrophilic layer helped the biocompatibility between the PDMS surface and the immobilization of the capture antibody. As a proof of concept for real applications, it was proposed the detection of CRP, a relevant biomarker of inflammation, by the MOPS approach. According the nature of the CRP, a sandwich immunoassay format is required which involved the use of a capture antibody and an additional detection antibody. The detection antibody should be biotynilated in order to be labelled for its recognition by a streptavidin-HRP conjugate. This conjugate is responsible of the generation of a colorimetric signal by the addition of a substrate solution. Therefore, in the presence of the CRP a positive signal will be recorded. The substrate solution that employed is based on a $\mathrm{TMB} / \mathrm{H}_{2} \mathrm{O}_{2}$ solution which in the presence of HRP a colored solution is obtained. As it can seen in Figure 5a, a buffer solution was initially injected at a constant flow of $100 \mu \mathrm{L} / \mathrm{min}$ using a Nemesys syringe pump (Cetoni GmbH, Germany) when a stable signal was achieved, a second Nemesys injected the substrate at a constant flow of $50 \mu \mathrm{L} / \mathrm{min}$. This provided with a stable colored response, which did not reach saturation. This is observed when the flow was stopped, the intensity dropped a significant value at all wavelengths. Finally, when the buffer solution was again injected, the signal was fully recovered. Figure $5 \mathrm{~b}$ shows the transient state of the absorbance vs wavelength between the MOPS filled with buffer (time zero) to the saturated signal of the substrate (time $>22 \mathrm{sec}$ ).

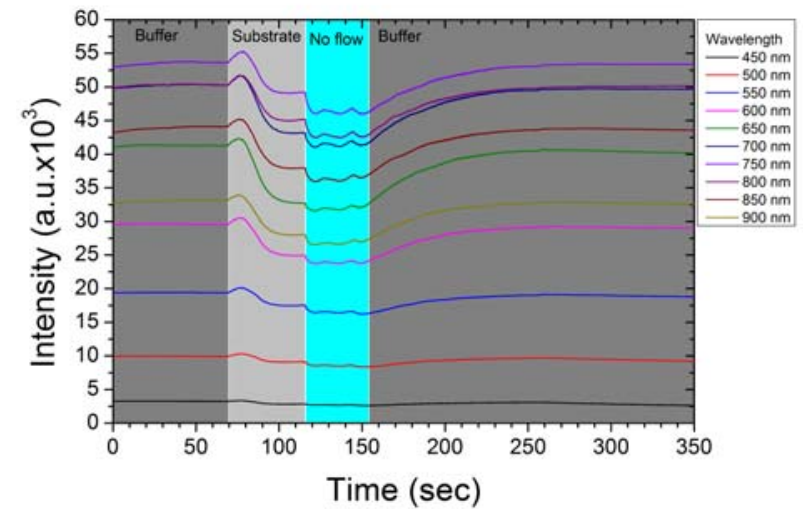

a)

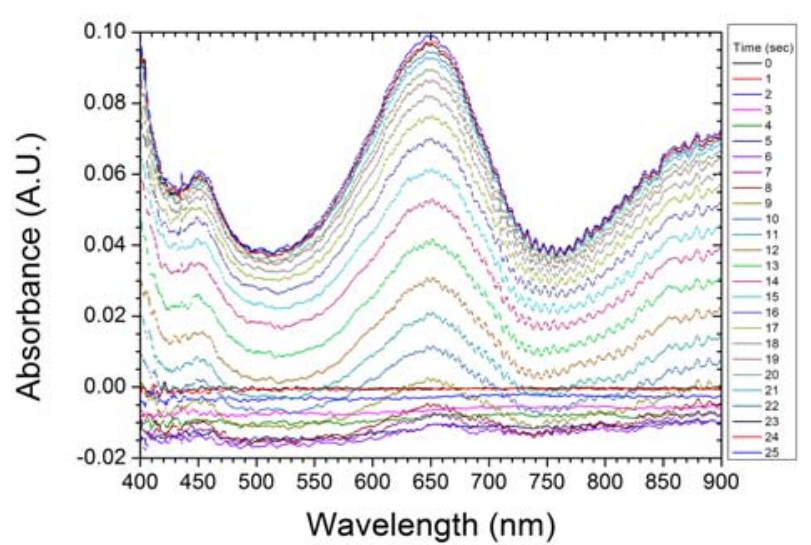

b)

Figure 5: a) Recorded intensity as a function of time for a CRP-functionalized MOPS with a concentration of $10 \mathrm{ng} / \mathrm{mL}$ when the substrate solution was injected. B) Absorbance as a function of the wavelength for different times, where the TMB spectral response is obtained.

\section{CONCLUSIONS}

We have reported a fully compatible modular optofluidic system. Its major asset is the capability of the system to be assembled in any configuration (based on the blocks introduced here). As a proof of concept they have been used for fluorescence measurements, showing that the inclusion of the absorbance filter module dramatically enhances the sensitivity. Similarly, in absorbance measurements, the inclusion of an additional module for expanding the optical path resulted in an increase of the sensitivity a factor 1.7 while decreasing the LoD from $5 \mu \mathrm{M}$ to $3.9 \mu \mathrm{M}$ using Quinoleine Yellow as analyte. Finally, the MOPS was biofunctionalized by specific antibodies against CRP and. Through complementary recognition, it was shown that a stable coloured response was obtained at a constant flow of $50 \mu \mathrm{L} / \mathrm{min}$. These results demonstrate the flexibility and applicability of the proposed approach. 


\section{ACKNOWLEDGEMENTS}

The work presented here is partly funded by the European Commission (LiPhos, Contract No. 317916).

\section{REFERENCES}

[1] Manz, A.; Graber, N.; Widmer, H. M, "Miniaturized total chemical analysis systems: a novel concept for chemical sensing". Sensors and actuators B: Chemical, 1 (1), 244-248, (1990).

[2] Wu, M.-H.; Huang, S.-B.; Lee, G.-B., "Microfluidic cell culture systems for drug research". Lab Chip 2010,10 (8), 939-956 (2010)

[3] Marcus, J. S.; Anderson, W. F.; Quake, S. R., "Parallel picoliter RT-PCR assays using microfluidics". Analytical Chemistry, 78 (3), 956-958 (2006).

[4] Menegatti, E.; Berardi, D.; Messina, M.; Ferrante, I.; Giachino, O.; Spagnolo, B.; Restagno, G.; Cognolato, L.; Roccatello, D., "Lab on a chip: Emerging analytical platforms for immune-mediated diseases". Autoimmunity Rev., 12 (8), 814-820 (2013)

[5] Shevkoplyas, S. S.; Yoshida, T.; Munn, L. L.; Bitensky, M. W., "Biomimetic autoseparation of leukocytes from whole blood in a microfluidic device". Analytical Chemistry, 77 (3), 933-937 (2005).

[6] Kimmel, D. W.; LeBlanc, G.; Meschievitz, M. E.; Cliffel, D. E., "Electrochemical Sensors and Biosensors. Analytical Chemistry, 84 (2), 685-707 (2012)

[7] Hoshino, K.; Huang, Y.-Y.; Lane, N.; Huebschman, M.; Uhr, J. W.; Frenkel, E. P.; Zhang, X., Microchip-based immunomagnetic detection of circulating tumor cells. Lab Chip 2011, 11 (20), 3449-3457.

[8] Balslev, S.; Jorgensen, A.; Bilenberg, B.; Mogensen, K. B.; Snakenborg, D.; Geschke, O.; Kutter, J. P.; Kristensen, A., "Lab-on-a-chip with integrated optical transducers". Lab Chip, 6 (2), 213-217 (2006).

[9] Llobera, A., Juvert, J., González-Fernández A., Ibarlucea B., Carregal-Romero E., Büttgenbach S., FernándezSánchez C., "Biofunctionalized All-Polymer Photonic Lab an a Chip With Integrated Solid-State Light Emitter" Light: Science \& Applications 4, e271(2015)

[10] Vila-Planas J, Fernández-Rosas E, Ibarlucea B, Demming S, Nogués C, Plaza JA, Domínguez C, Büttgenbach S, Llobera A, "Cell analysis using a multiple internal reflection photonic lab-on-a-chip". Nature protocols, 6(10), 1642-1655 (2011)

[11] Langelier S.M., Livak-Dahl E., Manzo A. J., Johnson B. N., Walter N. G. and Burns Mark A. "Flexible casting of modular self-aligning microfluidic assembly blocks" Lab Chip, 11, 1679-1687 (2011)

[12] Yuen P.K., Bliss J.T., Thompson C.C. and Peterson R.C. "Multidimensional modular microfluidic system"Lab Chip, 9, 3303-3305 (2009)

[13] Brammer M., Megnin C., Voigt A, Kohl M, and Mappes T. "Modular Optoelectronic Microfluidic Backplane for Fluid Analysis Systems" Journal of Microelectromech. Syst, 22(2):462-470, (2013).

[14] Xia YN, Whitesides GM, "Soft lithography” Angewandte Chemie-international edition, 37(5), 551-575 (1998)

[15]Bodas D, Khan-Malek C, "Hydrophilization and hydrophobic recovery of PDMS by oxygen plasma and chemical treatment - An SEM investigation”. Sensors and Actuators B-Chemical, 123(1), 368-373 (2007).

[16] Yu L, Li CM, Zhou Q, Luong JHT "Poly(vinyl alcohol) functionalized poly(dimethylsiloxane) solid surface for immunoassay". Bioconjugate Chemistry, 18(2), 281-284 (2007).

[17] Sandison ME, Cumming SA, Kolch W, Pitt AR, "On-chip immunoprecipitation for protein purification". Lab Chip, 10(20), 2805-2813 (2010)

[18] B. Ibarlucea, C. Fernández-Sánchez, S. Demming, S. Büttgenbach, A. Llobera "Selective Functionalisation of PDMS-Based Photonic Lab on a Chip For Biosensing" Analyst 136(17) 3496-3502 (2011).

[19] Ridker, P. M.; Buring, J. E.; Cook, N. R.; Rifai, N., "C-Reactive Protein, the Metabolic Syndrome, and Risk of Incident Cardiovascular Events". An 8-Year Follow-Up of 14719 Initially Healthy American Women, 107 (3), 391-397 (2003)

[20] Nacb Writing Group, M.; Morrow, D. A.; Cannon, C. P.; Jesse, R. L.; Newby, L. K.; Ravkilde, J.; Storrow, A. B.; Wu, A. H. B.; Christenson, R. H., "National Academy of Clinical Biochemistry Laboratory Medicine Practice Guidelines: Clinical Characteristics and Utilization of Biochemical Markers in Acute Coronary Syndromes". Circulation, 115 (13), e356-375 (2007)

[21] McDonnell, B.; Hearty, S.; Leonard, P.; O'Kennedy, R., "Cardiac biomarkers and the case for point-of-care testing”. Clinical Biochemistry, 42 (7-8), 549-561 (2009) 
[22]A. Llobera, R. Wilke, S. Büttgenbach "Enhancement of the Optical Response Of Poly(Dimethylsiloxane) Hollow Prisms" Talanta, 75, 473-479,(2008)

[23]X. Muñoz-Berbel, R. Rodríguez-Rodríguez, N. Vigués, J. Mas, S. Büttgenbach, E. Verpoorte, P. Ortiz and A. Llobera "Monolithically Integrated Biophotonic Lab on a Chip For Cell Retention, Proliferation and Simultaneous Monitoring Of Cell Metabolism” Lab chip 13(21), 4239-4247 (2013).

[24] Carregal-Romero E., Fernández-Sánchez C., Eguizabal A., Demming S., Büttgenbach S., Llobera A. "Development and Integration of Xerogel Polymeric Absorbance Micro-Filters nto Lab-On-Chip Systems" Opt. Exp. 20(21), 23700-23719,(2012) 